\title{
Does space make waste? The influence of ICU bed capacity on admission decisions
}

\author{
Esteban Mery' and Jeremy M Kahn*1-3 \\ University of Pittsburgh Department of Critical Care Medicine: Evidence-Based Medicine Journal Club, edited by Sachin Yende
}

\author{
Expanded abstract \\ Citation \\ Stelfox HT, Hemmelgarn BR, Bagshaw SM, Gao S, Doig \\ CJ, Nijssen-Jordan C, Manns B: Intensive care unit bed \\ availability and outcomes for hospitalized patients \\ with sudden clinical deterioration. Arch Intern Med \\ 2012, 172:467-474.
}

\section{Background}

Intensive care unit (ICU) beds are a scarce resource, and admissions may require prioritization when demand exceeds supply. However, there are few empiric data on whether the availability of ICU beds influences triage and processes of care for hospitalized patients who develop sudden clinical deterioration.

\section{Methods}

Objective: The objective was to evaluate the effect of ICU bed availability on the processes and outcomes of care for hospitalized patients with sudden clinical deterioration on a hospital ward.

Design: We conducted a retrospective cohort study.

Setting: The study was conducted in three hospitals in Calgary, Alberta, Canada, with 2,040 beds and a catchment population of 1.5 million individuals.

Subjects: Hospitalized adults $(\mathrm{n}=3,494)$ with a sudden clinical deterioration triggering medical emergency team (MET) activation between 1 January 2007 and 31 December 2009 participated.

Analysis: This study compared treatments and outcomes among sudden clinical deterioration patients according to the number of ICU beds available (zero, one, two, or more than two) at the time of the MET activation. The outcomes of interest were ICU admission rates (within 2 hours of MET activation), changes in the goals of care

*Correspondence: kahnjm@upmc.edu

'Department of Critical Care Medicine, University of Pittsburgh, 3550 Terrace

Street, Pittsburgh, PA 15221, USA

Full list of author information is available at the end of the article (resuscitative, medical, and comfort), and hospital mortality. All analyses were adjusted for hospital, physician, and patient factors.

\section{Results}

The cohort consisted of 3,494 patients. Reduced ICU bed availability was associated with a decreased likelihood of ICU admission within 2 hours of MET activation $(P=0.03)$ and with an increased likelihood of change in patient goals of care $(P<0.01)$. Patients with sudden clinical deterioration when zero ICU beds were available were $33.0 \%$ (95\% confidence interval (CI), $-5.1 \%$ to $57.3 \%)$ less likely to be admitted to the ICU and were $89.6 \%$ (95\% CI, $24.9 \%$ to $188.0 \%$ ) more likely to have their goals of care changed compared with when more than two ICU beds were available. However, hospital mortality did not vary significantly by ICU bed availability $(P=0.82)$.

\section{Conclusions}

For hospitalized patients with sudden clinical deterioration, ICU bed scarcity decreases the probability of ICU admission and increases the probability of initiating comfort measures on the ward but does not influence hospital mortality.

\section{Commentary}

In many hospitals, intensive care unit (ICU) beds are a scarce resource, necessitating sometimes difficult triage decisions [1]. Many factors can play a role in the decision to admit a patient to the ICU, including severity of illness and the need for specific treatments limited to the ICU (such as mechanical ventilation). However, these factors must be weighed subjectively, leading to wide variation in ICU admission rates across hospitals, even when severity of illness is controlled for [2].

Another factor that may influence the decision to admit a patient to the ICU is the availability of ICU beds themselves. Although, ideally, triage decisions would be based solely on patient- and system-level factors, it is likely that ICU clinicians make different decisions when there are fewer ICU beds available. Supporting this idea 
is evidence showing that countries with greater ICU bed availability typically admit patients with lower average illness severity [3]. However, ICU bed availability is only one of many differences between countries, and there are few data suggesting that ICU triage decisions depend on ICU bed availability at the hospital level.

To better understand this issue, Stelfox and colleagues [4] examined the association between ICU bed availability and processes and outcomes in 3,494 patients with a sudden clinical deterioration on the hospital ward over the course of a 2-year period. The study was performed in three hospitals in Alberta, Canada. The analysis was limited to patients for whom the sudden deterioration prompted medical emergency team (MET) activation. For each patient, the authors abstracted patient, physician, and hospital factors potentially relevant to ICU triage. Patient factors were demographic and socioeconomic variables as well as baseline goals of care (resuscitative, medical, or comfort care). Physician factors were the type of provider responding to the MET activation. Hospital factors were the number of ICU beds available (zero, one, two, or more than two), day of the week, and time of day of the MET activation. The authors then examined the relationship between these factors and the processes and outcomes of care following the MET activation.

Among the study's major findings was that MET activations when zero ICU beds were available tended to result in less intense care than MET activations in times of high bed availability ( $4.4 \%$ of patients were intubated when zero beds were available versus $10.1 \%$ when more than two ICU beds were available; $P<0.01$ ). In terms of processes of care, at times of high bed availability in the ICU, the patients were more likely to have arterial blood gas measurement, chest and abdominal radiographs, airway suctioning, and endotracheal intubation. In addition, when zero beds were available, patients were less likely to be admitted to the ICU (11.6\% versus $21.4 \%$; $P=0.03)$ and were more likely to have their goals of care changed to comfort $(14.9 \%$ versus $8.5 \% ; P \leq 0.01)$ as compared with when more than two beds were available. Despite this finding, ICU bed availability did not influence mortality, which ranged from $32 \%$ to $34 \%$ and did not statistically differ, regardless of the number of ICU beds available. These findings persisted after controlling for severity of illness and other patient-level factors during the MET activation.

The study has some limitations. Selection bias is a possibility if, at times of high bed availability, very sick patients could have been admitted directly to the ICU without the need for MET activation. Although the study was performed in three hospitals, all of them were part of a single publicly funded health region and these results may not generalize to other health systems.
Despite these limitations, this study strongly suggests that ICU bed availability, not just patient factors, influences the decision to admit a patient to the ICU. ICU bed availability was also associated with the decision to initiate comfort measures on the ward. Yet these decisions did not affect overall mortality. This finding suggests that, for a certain proportion of patients, admission to the ICU was simply delaying death rather than preventing it. Thus, it is likely that important decisions about whether to forgo life-sustaining treatment are influenced not just by patient preferences and conditions but also by the availability of resources. Better communication about care preferences at the end of life on hospital admission may have prevented some of these ICU admissions, both improving quality of care and reducing costs [5].

Overall, this study builds on the literature showing that ICU admission decisions are guided not only by patient factors but also by the availability of ICU beds [6]. Additionally, this study points to critical deficiencies in our ability to provide high-quality palliative care in the hospital. The decision to initiate comfort measures should be a patient-centered decision, based on patient preferences, family discussions, and severity of illness, not on ICU bed availability. Additional proactive efforts to address goals of care at hospital admission, rather than at the time of sudden clinical deterioration, are needed to ensure that our limited supply of ICU beds is used most effectively and efficiently.

\section{Recommendations}

ICU clinicians should evaluate their triage decisions and, if possible, routinely solicit patient preferences during medical emergencies, taking steps to ensure that ICU admission decisions are in line with the goals of the patient. Ultimately, these efforts will help ensure that scarce ICU resources are used most effectively and efficiently.

\section{Abbreviations}

ICU, intensive care unit; MET, medical emergency team.

\section{Competing interests}

The authors declare that they have no competing interests.

\section{Author details}

'Department of Critical Care Medicine, University of Pittsburgh, 3550 Terrace Street, Pittsburgh, PA 15221, USA. ${ }^{2}$ Clinical Research, Investigation, and Systems Modeling of Acute IIIness Center, Department of Critical Care Medicine, University of Pittsburgh School of Medicine, 3550 Terrace Street, Pittsburgh, PA 15221, USA. ${ }^{3}$ Department of Health Policy and Management, University of Pittsburgh Graduate School of Public Health, 3550 Terrace Street, Pittsburgh, PA 15221, USA.

Published: 8 May 2013

\section{References}

1. Sinuff T, Kahnamoui K, Cook DJ, Luce JM, Levy MM; Values Ethics and Rationing in Critical Care Task Force: Rationing critical care beds: a systemic review. Crit Care Med 2004, 32:1588-1597. 
2. Chen LM, Render M, Sales A, Kennedy EH, Wiitala W, Hofer TP: Intensive care unit admitting patterns in the Veterans affairs health care system. Arch Intern Med 2012, 172:1220-1226.

3. Wunsch H, Angus DC, Harrison DA, Collange O, Fowler R, Hoste EA, de Keizer NF, Kersten A, Linde-Zwirble WT, Sandiumenge A, Rowan KM: Variation in critical care services across North America and Western Europe. Crit Care Med 2008, 36:2787-2793.

4. Stelfox HT, Hemmelgarn BR, Bagshaw SM, Gao S, Doig CJ, Nijssen-Jordan C, Manns B: Intensive care unit bed availability and outcomes for hospitalized patients with sudden clinical deterioration. Arch Intern Med 2012, 172:467-474
5. White DB, Braddock CH 3rd, Bereknyei S, Curtis JR: Toward shared decision making at the end of life in intensive care units: opportunities for improvement. Arch Intern Med 2007, 167:461-467.

6. Strauss MJ, LoGerfo JP, Yeltatzie JA, Temkin N, Hudson LD: Rationing of intensive care unit services. An everyday occurrence. JAMA 1986, 255:1143-1146.

doi:10.1186/cc12688

Cite this article as: Mery E, Kahn JM: Does space make waste? The influence of ICU bed capacity on admission decisions. Critical Care 2013, 17:315. 